\title{
Vector Autoregressive Model of Wavelet Analysis and Granger Causality Positioning Epileptic EEG
}

\author{
Li Qiong ${ }^{1+}$, Zhang Lin ${ }^{2}$, Han $\mathrm{Xu}^{3}$, Fang $\mathrm{Li} \mathrm{Li}^{4}$, and Chen Liang ${ }^{5}$ \\ ${ }^{1}$ Mianyang Normal University, Mian Yang, China \\ ${ }^{2}$ Chengdu Yingde Bio-pharmaceutical Equipment and Technology Co. Ltd, Cheng du, China \\ ${ }^{3}$ Sichuan Airlines Co. Ltd, Cheng du, China \\ ${ }^{4}$ Patent Examination Cooperation Sichuan Center of Patent Office, Sipo, Sichuan Airlines Co. Ltd, Chengdu, \\ China \\ ${ }^{5}$ Chengdu Shuangliu International Airport Corporation Limited Mechanical and Electrical \\ Equipment Centre, Chengdu, China
}

\begin{abstract}
In this paper, we used the Granger causality test of vector autoregressive to study the connectivity of epileptic EEG. The real epilepsy EEG data show that Granger causality exists among epileptic lesions, which is conducive to understand the dynamic mode of operation of human brain at the system level, and to provide technical guidance for clinical diagnosis and treatment. And the results also show that using Granger causality of vector autoregressive to locate EEG epileptic wave has a bright future.
\end{abstract}

Keywords: epilepsy wave, EEG, wavelet analysis, vector autoregressive model, granger causality

\section{Introduction}

Epilepsy is a chronic brain dysfunction syndrome caused by various causes. In epileptic seizures, abnormal discharges of brain nerve cell populations cause brain dysfunction [1], and the information reflected by the EEG could not be provided by other physiological methods. Therefore, using computer to treat and analyze the EEG sigals in epilepsy patients is a valuable auxiliarytool for diagnosis, treatment, automatic detection of epilepsy and to reveal the pathogenesis of epilepsy [2].

Wavelet analysis is a new time-frequency analysis method developed in recent years. The traditional Fourier transform lacks spatial locality and can only determine the global property of singularity of a function, but it is difficult to determine the position and distribution of the singular point in space. On the basis of the traditional Fourier transform, wavelet analysis has both the spatial and frequency domain locality, so it becomes an effective tool to describe and seize the local properties of singularity [3]. Its variable time window and frequency window make it has a high adaptability to the signal, and it is very useful for extracting various characteristic information in biomedical signal. So it has a good positioning characteristics and analysis accuracy at sharp, sharp waves in epileptic EEG.

This paper introduce a new method using vector autoregressive model and Granger (Granger, 1969) causal relationship based on wavelet analysis[4] to locate epilepsy EEG information flow and to explore their connectivity. It can predict the location and seizure of epilepsy and indicate the direction of the follow-up treatment .

\section{Theory}

\footnotetext{
Corresponding author, Tel.: + 18608156868

E-mail address:5041342@qq.com.
} 


\subsection{AR Model}

The variables and error terms in the $\mathrm{p}$ order autoregressive model AR (p) are all transformed into vectors, and the Coefficient become Coefficient vector or matrix. We obtain a k-dimensional vector of p-order autoregressive model VAR (p):

$$
y_{t}=v+A_{1} y_{t-1}+A_{2} y_{t=2}+\cdots+A_{p} y_{t-p}+u_{t}
$$

$u[n]$ is a multivariate white noise, it's characteristic is

if $k=0$, then $u[n]=0$. Coefficient matrix

$$
E\left(\varepsilon_{t}\right)=0, \sum=\left[\begin{array}{cccc}
\sigma_{11}^{2} & \sigma_{21} & \cdots & \sigma_{k 1} \\
\sigma_{12} & \sigma_{22}^{2} & \cdots & \sigma_{k 2} \\
\vdots & \vdots & \ddots & \vdots \\
\sigma_{1 k} & \sigma_{2 k} & \cdots & \sigma_{k k}
\end{array}\right\rfloor ;
$$

$$
A_{i}=\left[\begin{array}{cccc}
a_{11 i} & a_{21 i} & \cdots & a_{k 1 i} \\
a_{12 i} & a_{22 i} & \cdots & a_{k 2 i} \\
\vdots & \vdots & \ddots & \vdots \\
a_{1 k i} & a_{2 k i} & \cdots & a_{k k i}
\end{array}\right], v=\left[\begin{array}{c}
v_{1} \\
v_{2} \\
\vdots \\
v_{k}
\end{array}\right\rfloor
$$

\subsection{Wavelet Analysis}

We denote the convolution of $f(x) \in L^{2}$ and $g(x) \in L^{2}$ as two functions

$$
f * g(x)=\int_{-\infty}^{\infty} f(u) g(x-u) d u
$$

$\theta(x)$ is a smoothing function, which should meet two conditions: $\int_{-\infty}^{+\infty} \theta(x) d x=1$ and $\lim _{|t| \rightarrow+\infty} \theta(x)=o$

Let $\varphi(x)=d \theta(x) / d x$, we can know $\varphi(x)$ satisfies the wavelet function permissibility condition, so it can be used as the wavelet mother function.

The following notation is introduced for arbitrary function $\theta(x)$ :

$$
\theta_{a}(x)=\frac{1}{a} \theta\left(\frac{x}{a}\right) \quad a>0
$$

Let $\varphi(x)$ be the wavelet function, $f(x)$ Convolution type wavelet transform at scale $a$ is defined as:

$$
W f(a, x)=f(x) * \varphi_{a}(x)=f(x) *\left(a \frac{d \theta_{a}}{d x}\right)(x)=a \frac{d}{d x}\left[f(x) * \theta_{a}(x)\right]
$$

It is known that the signal transition point is related to the wavelet transform modulus maxima. We have the following definitions:

Definition 1. The continuous wavelet transform of signal $\mathrm{f}(\mathrm{x})$ is defined as:

$$
W_{a} f(a, x)=\frac{1}{|a|^{1 / 2}} \int_{R} f(t) \varphi\left(\frac{t-x}{a}\right) d t
$$

among them, $a$ is the scale, $\varphi\left(\frac{t-x}{a}\right)$ is a wavelet function.

Definition 2. At scale $a_{0}$, if $\frac{d}{d x} W f(a, x)$ has a zero-crossing point at $x=x_{0}$, we call $\left(a_{0}, x_{0}\right)$ is a local mutation point.

Definition 3. For $\forall x \in\left(x_{0}, \delta\right)$, we have $\left|W f\left(a_{0}, x\right)\right| \leq\left|W f\left(a_{0}, x_{0}\right)\right|$, and we call $\left(a_{0}, x_{0}\right)$ the maximum modulus [5]

In summary, when the first derivative of smoothing function $\varphi(x)$ as the wavelet function of the wavelet transform for the signal, the wavelet transform The position of the first derivative modulus maxima at each scale corresponds to the position of the discontinuity of the signal. 


\subsection{Granger Causality}

Granger causality (Granger, 1969) is derived from a concept in economics, which is mainly using time series to identify causal relationships. It pointe out that if a time series y can cause (or influence) another time series $\mathrm{x}$, and $\mathrm{y}$ information can predict the future value of $\mathrm{x}$, then a causal relationship can be predicted. More specifically, there are two discrete time series $\mathrm{x}$ and $\mathrm{y}$, if we use the past $\mathrm{x}$ and $\mathrm{y}$ ' $\mathrm{s}$ value to predict the effect of the current $x$ value alone, it is more effctive than using the value of the past $x$ to predict the current $\mathrm{x}$ value, Then we think that there is Granger causality between $\mathrm{y}$ and $\mathrm{x}$. On this basis, the time structure between $\mathrm{x}$ and $\mathrm{y}$ is used to determine the direct effects that may exist between them.

\subsection{Effective Connectivity}

Geweke (1982) [6] proposed a linear dependence between $\mathrm{x}$ and $\mathrm{y}$ time series. This scale is based on the vector autoregressive model and the Granger causality concept [7]. $F_{x, y}$ is defined as follows:

$$
F_{x, y}=F_{x \rightarrow y}+F_{y \rightarrow x}+F_{x \cdot y}
$$

$F_{x \rightarrow y}$ is a measure of the direct impact of $\mathrm{x}$ to $\mathrm{y}$, and if past $\mathrm{x}$ values can predict the current value of $\mathrm{y}$, then $F_{x \rightarrow y}>0$ 。 The same definition $F_{y \rightarrow x}$ 。 $F_{x \cdot y}$ quantifies the current x (y) value, predicting the current $\mathrm{x}(\mathrm{y})$ value by the current value of $\mathrm{y}(\mathrm{x})$ and the value of past $\mathrm{x}(\mathrm{y})$.

\section{Dealing with Real Data and Analysis Results}

We used this method in the clinical measured EEG signal, from which a signal is intercepted for analysis Figure 1 is the interception of the epileptic EEG signal. This data extracted by wavelet analysis of the epileptic focus [8] results in the following reconstruction of Figure 2. According to Fig. 2, we can find that it is very easy and clear to locate the spike, sharp and slow wave signals in EEG data by using the wavelet analysis.

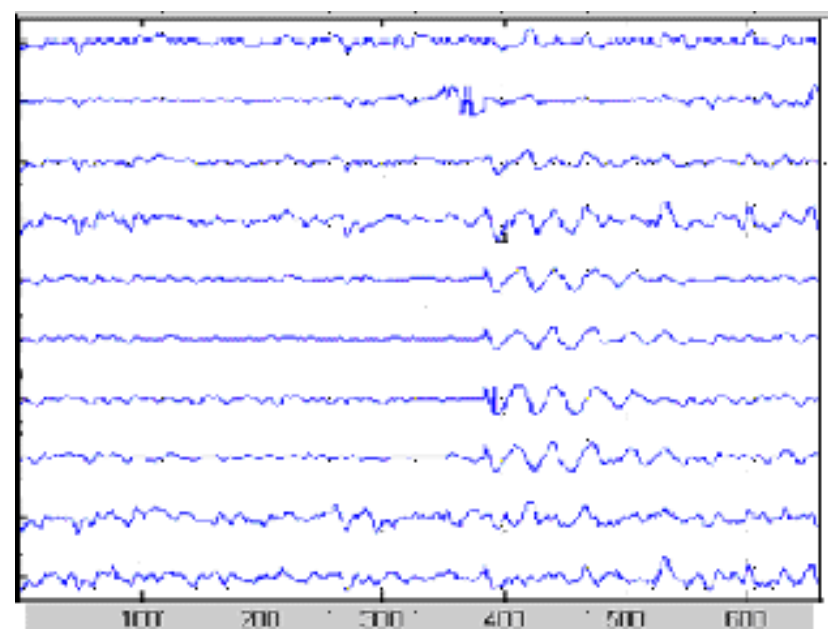

Fig. 1: EEG signals of epilepsy.

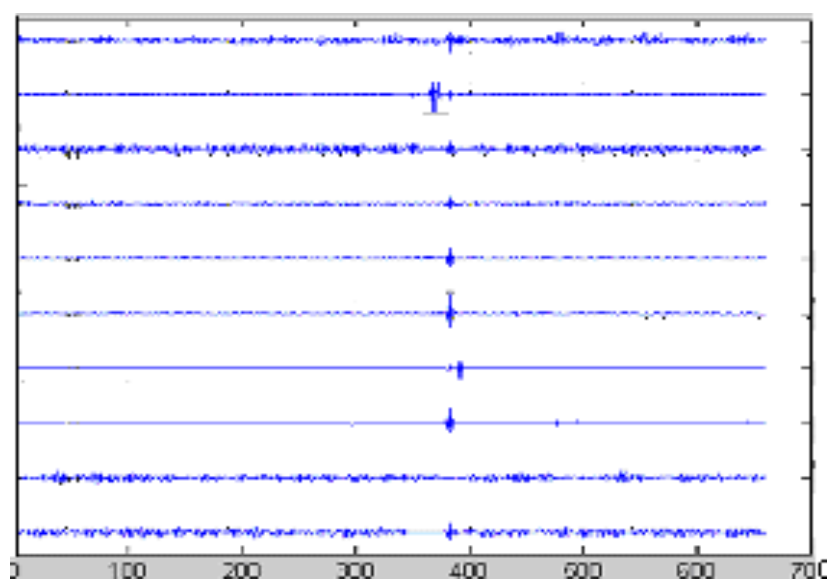

Fig. 2: The singular value of epileptic EEG obtained by wavelet analysis. 
Granger causality analysis of the autoregressive model of the signals in Fig. 1 and Fig. 2, shows the influence of each other. Processing the relationship between the 6th signal and the 7th signal, Let the 6th signa be the time series $\mathrm{x}$, And the 7 th signal as the time series $\mathrm{y}$, The resulting $F_{x, y}=F_{x \rightarrow y}+F_{y \rightarrow x}+F_{x \cdot y}$ relationship is shown in Figure 1. According to the statistics of normal distribution of the data in the table, the 6th signal has a direct influence on the 7th signal, that is, the information flow is from the 6th signal to the 7th channel.

Table 1: Connectivity Analysis of 6th and 7th Signals of Raw and Wavelet Processing

\begin{tabular}{|l|l|l|l|l|}
\hline & $F_{x, y}$ & $F_{x \rightarrow y}$ & $F_{y \rightarrow x}$ & $F_{x \cdot y}$ \\
\hline The original 6th and 7th singal & 0.3424 & 0.1013 & 0.0641 & 0.1770 \\
\hline The 6th and 7th signal rocessed by wavelet & 0.1070 & 0.0026 & 0.0003 & 0.1041 \\
\hline
\end{tabular}

\section{Conclusion}

EEG is an effective basis for the diagnosis of epilepsy,based on the singularity detection of signal by wavelet analysis, the Granger causality of vector autoregressive model was used to locate epileptic EEG.The results show that the results of wavelet analysis are more effective than the Granger causality of vector autoregressive model in detecting epileptic signal connectivity, which is helpful for clinicians to observe and analyze EEG more accurately and improve epilepsy Diagnostic accuracy.

Computer-aided disease treatment has been widely applied to modern medicine, the algorithm for nonstationary, non-linear and other variability of larger biomedical signal classification and analysis, can more effectively provide more help to clinical diagnosis It is worthy for the further study of application prospect in EEG and other biomedical signal analysis and process.

\section{References}

[1] Xiaowei Tang. Brain functional imaging [M]. China University of Science and Technology Press, 1999.10

[2] L. B. Good. S. Sabesan. S. T. Marsh. K. S. Tsakalis and L. D. lasemidis. Control of synchronization of brain dynamics leads to control of epileptic seizures in rodents. Int. J. Neural Syst.19(3) (2009) 173-196

[3] Changhua Hu, Junbo Zhang, Jun Xia, Wei Zhang. System Analysis and Design Based on MATLAB - Wavelet Analysis[M]. Xi'an: Xi'an University of Electronic Science and Technology Press, 1999. 12

[4] Grang er C W $1 \mathrm{~J} 1$ Investiag ting causa 1 relationsby econom etric $\mathrm{m}$ od els and cross - sp ectra $1 \mathrm{~m}$ ethod s. E conom etrica, 1969, 37: $424-438$

[5] Huafu Chen, Shouming Zhong, Dezhong Yao. Detection singularity value of character wave in epileptic EEG by wavelet, Proceedings of IEEE-PRESS 2002 Conference. 1095

[6] Geweke J. Measurement of linear dependence and feedback between multiple time series. J Am Stat Assoc 1982; 77: 304-13.

[7] Goebel, R., Roebroeck, A., et al., 2003. Investigating directed cortical interactions in time-resolved fMRI data using vector autoregressive modeling and Granger causality mapping. Magn. Reson. Imaging 21(10), 1251- 1261.

[8] Lin Zhang, Ling Zeng, Wei Liao etc. Comparative Study of Wavelet Analysis and Independent Component Analysis of Epileptic EEG Signals. Chinese Journal of Biomedical Engineering, 2007, 269-272 